\title{
On Algebras of Algorithms and Specifications over Uninterpreted Data
}

\author{
Ievgen Ivanov \\ Taras Shevchenko National University \\ Kyiv, Ukraine
}

\author{
Artur Korniłowicz (iD \\ Institute of Informatics \\ University of Białystok \\ Poland
}

\author{
Mykola Nikitchenkc \\ Taras Shevchenko National University \\ Kyiv, Ukraine
}

\begin{abstract}
Summary. This paper continues formalization in Mizar [2, 1] of basic notions of the composition-nominative approach to program semantics [13] which was started in 8, 11.

The composition-nominative approach studies mathematical models of computer programs and data on various levels of abstraction and generality and provides tools for reasoning about their properties. Besides formalization of semantics of programs, certain elements of the composition-nominative approach were applied to abstract systems in a mathematical systems theory [4, 6, 7, 5, 3].

In the paper we introduce a definition of the notion of a binominative function over a set $D$ understood as a partial function which maps elements of $D$ to $D$. The sets of binominative functions and nominative predicates 11] over given sets form the carrier of the generalized Glushkov algorithmic algebra [14. This algebra can be used to formalize algorithms which operate on various data structures (such as multidimensional arrays, lists, etc.) and reason about their properties.

We formalize the operations of this algebra (also called compositions) which are valid over uninterpretated data and which include among others the sequential composition, the prediction composition, the branching composition, the monotone Floyd-Hoare composition, and the cycle composition. The details on formalization of nominative data and the operations of the algorithmic algebra over them are described in [10, 12, 9].
\end{abstract}

MSC: 68Q60 68T37 03B70 03B35

Keywords: Glushkov algorithmic algebra; uninterpreted data

MML identifier: PARTPR_2, version: 8.1.08 5.53.1335 


\section{Preliminaries}

From now on $x$ denotes an object and $n$ denotes a natural number.

Let $X, Y$ be sets. Observe that every element of $X \dot{\rightarrow} Y$ is $X$-defined and every element of $X \dot{\rightarrow} Y$ is $Y$-valued.

Now we state the proposition:

(1) Let us consider sets $X, Y, Z, T$, objects $x, y, z$, and a function $f$ from $X \times Y \times Z$ into $T$. Suppose $x \in X$ and $y \in Y$ and $z \in Z$ and $T \neq \emptyset$. Then $f(x, y, z) \in T$.

One can verify that there exists a set which is non empty and has not non empty elements.

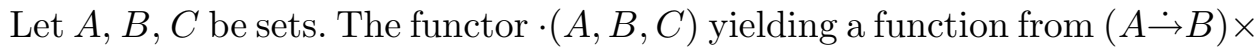
$(B \dot{\rightarrow} C)$ into $A \dot{\rightarrow} C$ is defined by

(Def. 1) for every partial function $f$ from $A$ to $B$ and for every partial function $g$ from $B$ to $C, i t(f, g)=g \cdot f$.

From now on $D$ denotes a non empty set and $p, q$ denote partial predicates of $D$.

Now we state the propositions:

(2) If $q$ is total, then $\operatorname{dom} p \subseteq \operatorname{dom}(p \vee q)$.

(3) If $q$ is total, then $\operatorname{dom} p \subseteq \operatorname{dom}(p \wedge q)$.

(4) If $q$ is total, then $\operatorname{dom} p \subseteq \operatorname{dom}(p \Rightarrow q)$.

2. Operations in Algebras of Algorithms and Specifications over UNINTERPRETED DATA

From now on $D$ denotes a set.

Let us consider $D$. The functor $\operatorname{FPrg}(D)$ yielding a set is defined by the term (Def. 2) $D \dot{\rightarrow} D$.

Observe that $\operatorname{FPrg}(D)$ is non empty and functional.

A binominative function of $D$ is a partial function from $D$ to $D$. Now we state the proposition:

(5) Let us consider a non empty set $D$, and a binominative function $f$ of $D$. Then $\operatorname{id}_{\text {field } f}$ is a binominative function of $D$.

In the sequel $p, q$ denote partial predicates of $D$ and $f, g$ denote binominative functions of $D$.

Let us consider $D$ and $p$. Let $F$ be a function from $\operatorname{Pr}(D)$ into $\operatorname{Pr}(D)$. One can check that $F(p)$ is function-like and relation-like. 
Let $p$ be an element of $\operatorname{Pr}(D)$. One can check that $F(p)$ is function-like and relation-like.

Let us consider $p$ and $q$. Let $F$ be a function from $\operatorname{Pr}(D) \times \operatorname{Pr}(D)$ into $\operatorname{Pr}(D)$. Observe that $F(p, q)$ is function-like and relation-like.

Let $p, q$ be elements of $\operatorname{Pr}(D)$. One can check that $F(p, q)$ is function-like and relation-like.

Let $x$ be an element of $\operatorname{Pr}(D) \times \operatorname{Pr}(D)$. Observe that $F(x)$ is function-like and relation-like.

Let us consider $f$. Let $F$ be a function from $\operatorname{FPrg}(D)$ into $\operatorname{FPrg}(D)$. Let us observe that $F(f)$ is function-like and relation-like.

Let us consider $p$ and $g$. Let $F$ be a function from $\operatorname{Pr}(D) \times \mathrm{FPrg}(D) \times \operatorname{FPrg}(D)$ into $\operatorname{FPrg}(D)$. One can check that $F(p, f, g)$ is function-like and relation-like and $F(\langle p, f, g\rangle)$ is function-like and relation-like.

Let us consider $q$. Let $F$ be a function from $\operatorname{Pr}(D) \times \operatorname{FPrg}(D) \times \operatorname{Pr}(D)$ into $\operatorname{Pr}(D)$. One can check that $F(p, f, q)$ is function-like and relation-like and $F(\langle p$, $f, q\rangle)$ is function-like and relation-like.

Let $D$ be a set. We introduce the notation $\operatorname{id}_{\mathrm{PP}}(D)$ as a synonym of $\operatorname{id}_{D}$.

One can verify that the functor $\operatorname{id}_{\mathrm{PP}}(D)$ yields a binominative function of $D$. Let $D$ be a non empty set and $d$ be an element of $D$. The functor $\operatorname{id}_{\mathrm{PP}}(d)$ yielding an element of $D$ is defined by the term

(Def. 3) $\operatorname{id}_{\mathrm{PP}}(D)(d)$.

Let us consider $D$. The functor $\bullet(D)$ yielding a function from $\operatorname{FPrg}(D) \times$ $\operatorname{FPrg}(D)$ into $\operatorname{FPrg}(D)$ is defined by the term

(Def. 4) $\cdot(D, D, D)$.

Let us consider $D, f$, and $g$. The functor $f \bullet g$ yielding a binominative function of $D$ is defined by the term

$($ Def. 5$) \bullet(D)(f, g)$.

Let us consider $D$. The functor $\cdot(D)$ yielding a function from $\operatorname{FPrg}(D) \times$ $\operatorname{Pr}(D)$ into $\operatorname{Pr}(D)$ is defined by the term

(Def. 6) $\cdot(D, D$, Boolean $)$.

Let us consider $D, f$, and $p$. The functor $f \cdot p$ yielding a partial predicate of $D$ is defined by the term

(Def. 7$) \cdot(D)(f, p)$.

Let $F$ be a function from $\operatorname{Pr}(D) \times \mathrm{FPrg}(D) \times \mathrm{FPrg}(D)$ into $\mathrm{FPrg}(D), p$ be a partial predicate of $D$, and $f, g$ be binominative functions of $D$. One can check that $F(p, f, g)$ is function-like and relation-like.

Now we state the proposition: 
(6) If $x \in \operatorname{dom}(f \cdot p)$, then $x \in \operatorname{dom}(p \cdot f)$ and $((p \cdot f)(x)=$ true or $(p \cdot f)(x)=$ false).

The scheme PredToNomPredEx deals with a set $\mathcal{D}$ and a set $D_{1}$ and a unary predicate $\mathcal{P}$ and states that

(Sch. 1) There exists a partial predicate $p$ of $\mathcal{D}$ such that $\operatorname{dom} p=D_{1}$ and for every object $d$ such that $d \in \operatorname{dom} p$ holds if $\mathcal{P}[d]$, then $p(d)=$ true and if not $\mathcal{P}[d]$, then $p(d)=$ false

provided

- $D_{1} \subseteq \mathcal{D}$.

The scheme PredToNomPredUnique deals with a set $\mathcal{D}$ and a set $D_{1}$ and a unary predicate $\mathcal{P}$ and states that

(Sch. 2) For every partial predicates $p, q$ of $\mathcal{D}$ such that $\operatorname{dom} p=D_{1}$ and for every object $d$ such that $d \in \operatorname{dom} p$ holds if $\mathcal{P}[d]$, then $p(d)=$ true and if not $\mathcal{P}[d]$, then $p(d)=$ false and $\operatorname{dom} q=D_{1}$ and for every object $d$ such that $d \in \operatorname{dom} q$ holds if $\mathcal{P}[d]$, then $q(d)=$ true and if not $\mathcal{P}[d]$, then $q(d)=$ false holds $p=q$.

Let us consider $D$. The functor isEmpty $(D)$ yielding a partial predicate of $D$ is defined by

(Def. 8) $\operatorname{dom} i t=D$ and for every object $d$ such that $d \in \operatorname{dom} i t$ holds if $d=\emptyset$, then it $(d)=$ true and if $d \neq \emptyset$, then it $(d)=$ false.

Let $D$ be a set with non non empty elements. The functor Empty ${ }_{D}$ yielding a binominative function of $D$ is defined by the term

(Def. 9) $D \longmapsto \emptyset$.

Let us consider $D$. The functor $\perp_{D}$ yielding a binominative function of $D$ is defined by the term

(Def. 10) $\emptyset$.

In the sequel $D$ denotes a non empty set, $p, q$ denote partial predicates of $D$, and $f, g, h$ denote binominative functions of $D$.

Let us consider $D$. The functor $\operatorname{IF}(D)$ yielding a function from $\operatorname{Pr}(D) \times$ $\operatorname{FPrg}(D) \times \operatorname{FPrg}(D)$ into $\operatorname{FPrg}(D)$ is defined by

(Def. 11) for every partial predicate $p$ of $D$ and for every binominative functions $f$, $g$ of $D$, dom it $(p, f, g)=\{d$, where $d$ is an element of $D: d \in \operatorname{dom} p$ and $p(d)=$ true and $d \in \operatorname{dom} f$ or $d \in \operatorname{dom} p$ and $p(d)=$ false and $d \in \operatorname{dom} g\}$ and for every object $d$, if $d \in \operatorname{dom} p$ and $p(d)=$ true and $d \in \operatorname{dom} f$, then it $(p, f, g)(d)=f(d)$ and if $d \in \operatorname{dom} p$ and $p(d)=$ false and $d \in \operatorname{dom} g$, then $i t(p, f, g)(d)=g(d)$. 
Let us consider $D, p, f$, and $g$. The functor $\operatorname{IF}(p, f, g)$ yielding a binominative function of $D$ is defined by the term

(Def. 12) $\operatorname{IF}(D)(p, f, g)$.

Now we state the proposition:

(7) Suppose $x \in \operatorname{dom}(\operatorname{IF}(p, f, g))$. Then

(i) $x \in \operatorname{dom} p$ and $p(x)=$ true and $x \in \operatorname{dom} f$, or

(ii) $x \in \operatorname{dom} p$ and $p(x)=$ false and $x \in \operatorname{dom} g$.

Let us consider $D$. The functor $\operatorname{FH}(D)$ yielding a function from $\operatorname{Pr}(D) \times$ $\mathrm{FPrg}(D) \times \operatorname{Pr}(D)$ into $\operatorname{Pr}(D)$ is defined by

(Def. 13) for every partial predicates $p, q$ of $D$ and for every binominative function $f$ of $D, \operatorname{dom} i t(p, f, q)=\{d$, where $d$ is an element of $D: d \in \operatorname{dom} p$ and $p(d)=$ false or $d \in \operatorname{dom}(q \cdot f)$ and $(q \cdot f)(d)=$ true or $d \in \operatorname{dom} p$ and $p(d)=$ true and $d \in \operatorname{dom}(q \cdot f)$ and $(q \cdot f)(d)=$ false $\}$ and for every object $d$, if $d \in \operatorname{dom} p$ and $p(d)=$ false or $d \in \operatorname{dom}(q \cdot f)$ and $(q \cdot f)(d)=$ true, then it $(p, f, q)(d)=$ true and if $d \in \operatorname{dom} p$ and $p(d)=$ true and $d \in \operatorname{dom}(q \cdot f)$ and $(q \cdot f)(d)=$ false, then $i t(p, f, q)(d)=$ false.

Let us consider $D, p, q$, and $f$. The functor $\operatorname{FH}(p, f, q)$ yielding a partial predicate of $D$ is defined by the term

(Def. 14) $\mathrm{FH}(D)(p, f, q)$.

Now we state the proposition:

(8) Suppose $x \in \operatorname{dom}(\mathrm{FH}(p, f, q))$. Then

(i) $x \in \operatorname{dom} p$ and $p(x)=$ false, or

(ii) $x \in \operatorname{dom}(q \cdot f)$ and $(q \cdot f)(x)=$ true, or

(iii) $x \in \operatorname{dom} p$ and $p(x)=$ true and $x \in \operatorname{dom}(q \cdot f)$ and $(q \cdot f)(x)=$ false.

Let us consider $D$. The functor $\mathrm{WH}(D)$ yielding a function from $\operatorname{Pr}(D) \times$ $\operatorname{FPrg}(D)$ into $\operatorname{FPrg}(D)$ is defined by

(Def. 15) for every partial predicate $p$ of $D$ and for every binominative function $f$ of $D$, dom $i t(p, f)=\{d$, where $d$ is an element of $D$ : there exists a natural number $n$ such that for every natural number $i$ such that $i \leqslant n-1$ holds $d \in \operatorname{dom}\left(p \cdot\left(f^{i}\right)\right)$ and $\left(p \cdot\left(f^{i}\right)\right)(d)=$ true and $d \in \operatorname{dom}\left(p \cdot\left(f^{n}\right)\right)$ and $\left(p \cdot\left(f^{n}\right)\right)(d)=$ false $\}$ and for every object $d$ such that $d \in \operatorname{dom} i t(p, f)$ there exists a natural number $n$ such that for every natural number $i$ such that $i \leqslant n-1$ holds $d \in \operatorname{dom}\left(p \cdot\left(f^{i}\right)\right)$ and $\left(p \cdot\left(f^{i}\right)\right)(d)=$ true and $d \in \operatorname{dom}\left(p \cdot\left(f^{n}\right)\right)$ and $\left(p \cdot\left(f^{n}\right)\right)(d)=$ false and it $(p, f)(d)=\left(f^{n}\right)(d)$.

Let us consider $D, p$, and $f$. The functor $\mathrm{WH}(p, f)$ yielding a binominative function of $D$ is defined by the term

(Def. 16) $\mathrm{WH}(D)(p, f)$. 
The functor $\sim D$ yielding a function from $\operatorname{Pr}(D)$ into $\operatorname{Pr}(D)$ is defined by

(Def. 17) for every partial predicate $p$ of $D, \operatorname{dom}(i t(p))=\{d$, where $d$ is an element of $D: d \notin \operatorname{dom} p\}$ and for every element $d$ of $D$ such that $d \notin \operatorname{dom} p$ holds it $(p)(d)=$ true.

Let $D$ be a non empty set and $p$ be a partial predicate of $D$. The functor $\sim p$ yielding a partial predicate of $D$ is defined by the term

(Def. 18) $\quad(\sim D)(p)$.

Now we state the propositions:

(9) Let us consider an element $d$ of $D$. Then $d \in \operatorname{dom} p$ if and only if $d \notin$ $\operatorname{dom}(\sim p)$.

(10) If $p$ is total, then $\operatorname{dom}(\sim p)=\emptyset$.

\section{REFERENCES}

[1] Grzegorz Bancerek, Czesław Byliński, Adam Grabowski, Artur Korniłowicz, Roman Matuszewski, Adam Naumowicz, and Karol Pąk. The role of the Mizar Mathematical Library for interactive proof development in Mizar. Journal of Automated Reasoning, 61(1):9-32, 2018. do1 $10.1007 /$ s10817-017-9440-6

[2] Adam Grabowski, Artur Korniłowicz, and Adam Naumowicz. Four decades of Mizar. Journal of Automated Reasoning, 55(3):191-198, 2015. doi $10.1007 / \mathrm{s} 10817-015-9345-1$

[3] Ievgen Ivanov. On the underapproximation of reach sets of abstract continuous-time systems In Erika Ábrahám and Sergiy Bogomolov, editors, Proceedings 3rd International Workshop on Symbolic and Numerical Methods for Reachability Analysis, SNR@ETAPS 2017, Uppsala, Sweden, 22nd April 2017, volume 247 of EPTCS, pages 46-51, 2017. doi: $10.4204 /$ EPTCS.247.4.

[4] Ievgen Ivanov. On representations of abstract systems with partial inputs and outputs. In T. V. Gopal, Manindra Agrawal, Angsheng Li, and S. Barry Cooper, editors, Theory and Applications of Models of Computation - 11th Annual Conference, TAMC 2014, Chennai, India, April 11-13, 2014. Proceedings, volume 8402 of Lecture Notes in Computer Science, pages 104-123. Springer, 2014. ISBN 978-3-319-06088-0. doi 10.1007/978-3-319-06089-7_8.

[5] Ievgen Ivanov. On local characterization of global timed bisimulation for abstract continuous-time systems. In Ichiro Hasuo, editor, Coalgebraıc Methods in Computer Scıence - 13th IFIP WG 1.3 International Workshop, CMCS 2016, Colocated with ETAPS 2016, Eindhoven, The Netherlands, April 2-3, 2016, Revised Selected Papers, volume 9608 of Lecture Notes in Computer Science, pages 216-234. Springer, 2016. ISBN 978-3319-40369-4. doi 10.1007/978-3-319-40370-0_13.

[6] Ievgen Ivanov, Mykola Nikitchenko, and Uri Abraham. On a decidable formal theory for abstract continuous-time dynamical systems. In Vadim Ermolayev, Heinrich C. Mayr, Mykola Nikitchenko, Aleksander Spivakovsky, and Grygoriy Zholtkevych, editors, Information and Communication Technologies in Education, Research, and Industrial Applications: 10th International Conference, ICTERI 2014, Kherson, Ukraine, June 9-12, 2014, Revised Selected Papers, pages 78-99. Springer International Publishing, 2014. ISBN 978-3-319-13206-8. doi 10.1007/978-3-319-13206-8_4.

[7] Ievgen Ivanov, Mykola Nikitchenko, and Uri Abraham. Event-based proof of the mutual exclusion property of Peterson's algorithm. Formalized Mathematics, 23(4):325-331, 2015. doi: $10.1515 /$ forma-2015-0026.

[8] Ievgen Ivanov, Mykola Nikitchenko, Andrii Kryvolap, and Artur Korniłowicz. Simplenamed complex-valued nominative data - definition and basic operations. Formalized Mathematics, 25(3):205-216, 2017. doi 10.1515/forma-2017-0020.

[9] Ievgen Ivanov, Artur Korniłowicz, and Mykola Nikitchenko. Implementation of the 
composition-nominative approach to program formalization in Mizar. The Computer Science Journal of Moldova, 26(1):59-76, 2018.

[10] Artur Kornilowicz, Andrii Kryvolap, Mykola Nikitchenko, and Ievgen Ivanov. Formalization of the algebra of nominative data in Mizar In Maria Ganzha, Leszek A. Maciaszek, and Marcin Paprzycki, editors, Proceedings of the 2017 Federated Conference on Computer Science and Information Systems, FedCSIS 2017, Prague, Czech Republic, September 3-6, 2017., pages 237-244, 2017. ISBN 978-83-946253-7-5. doi 10.15439/2017F301

[11] Artur Korniłowicz, Ievgen Ivanov, and Mykola Nikitchenko. Kleene algebra of partial predicates. Formalized Mathematics, 26(1):11-20, 2018. doi:10.2478/forma-2018-0002

[12] Artur Korniłowicz, Andrii Kryvolap, Mykola Nikitchenko, and Ievgen Ivanov. Formalization of the nominative algorithmic algebra in Mizar. In Jerzy Świątek, Leszek Borzemski, and Zotia Wilimowska, editors, Information Systems Architecture and Technology: Proceedings of 38th International Conference on Information Systems Architecture and Technology - ISAT 2017: Part II, pages 176-186. Springer International Publishing, 2018. ISBN 978-3-319-67229-8. doi 10.1007/978-3-319-67229-8_16.

[13] Nikolaj S. Nikitchenko. A composition nominative approach to program semantics. Technical Report IT-TR 1998-020, Department of Information Technology, Technical University of Denmark, 1998.

[14] Volodymyr G. Skobelev, Mykola Nikitchenko, and Ievgen Ivanov. On algebraic properties of nominative data and functions. In Vadim Ermolayev, Heinrich C. Mayr, Mykola N1kitchenko, Aleksander Spivakovsky, and Grygoriy Zholtkevych, editors, Information and Communication Technologies in Education, Research, and Industrial Applications - 10th International Conference, ICTERI 2014, Kherson, Ukraine, June 9-12, 2014, Revised Selected Papers, volume 469 of Communications in Computer and Information Science, pages 117-138. Springer, 2014. ISBN 978-3-319-13205-1. doi 10.1007/978-3-319-13206-8_6.

Accepted June 29, 2018 